\title{
Acumulação de Capital, Abertura Financeira e Endividamento Externo: um Modelo Macrodinâmico Pós-Keynesiano com Câmbio Flexível e Mobilidade de Capitais
}

\section{Capital Accumulation, Financial Opening and External Indebtedness: a Post-Keynesian Macrodynamic Model with Floating Exchange Rate and Capital Mobility}

Flávio Augusto Corrêa Basilio*

José Luís Oreiro**

Resumo: Neste artigo apresentamos um modelo macrodinâmico de inspiração pós-keynesiana com o objetivo de analisar os efeitos da abertura da conta de capitais sobre a performance macroeconômica das economias em desenvolvimento. Nesse particular, os exercícios de estática comparativa mostram que um aumento do endividamento externo pode ter efeitos benéficos para a economia em curto prazo, uma vez que induz o aumento do grau de utilização da capacidade produtiva e da taxa de crescimento do estoque de capital. No entanto, a dinâmica de longo prazo mostra que, sob certas condições, o acesso ao mercado internacional de capital pode gerar trajetórias explosivas para o sistema econômico, notadamente num contexto em que a política monetária é conduzida com base num regime de metas de inflação. Além disso, demonstra-se que uma redução da meta de inflação perseguida pelo Banco Central pode gerar um aumento permanente da taxa real de juros, bem como um aumento do endividamento externo da economia, contribuindo, assim, para uma maior fragilidade externa.

Palavras-chave: Crescimento endógeno. Poupança externa. Câmbio flexível.

\begin{abstract}
The objective of this article is to present a post-keynesian macrodynamic model in order to analyze the effects of the liberalization of capital account over the economic performance of developing economies. In this setting, comparative static analysis show that an increase in the level of external debt can have positive effects for the economy in the short-run, since it induces an increase in the level of capacity utilization and the growth rate of capital stock. However, long-run dynamics show that, under certain conditions, the access to international capital markets can generate explosive paths for the economic system, mainly if monetary policy is conducted under a Inflation Targeting Regime. Besides that, it was show that a reduction in the target rate of inflation can generate a permanent increase in the level of interest rate as well as

* Doutor em Economia pela Universidade de Brasília (UnB). Professor do Centro Universitário UDF E-mail: flaviobasilio@gmail.com

* * Doutor em Economia pela Universidade Federal do Rio de Janeiro (UFRJ). Professor do Instituto de Economia da Universidade Federal do Rio de Janeiro (UFRJ). Pesquisador nível IB do CNPq e Presidente da Associação Keynesiana Brasileira. E-mail: jose.oreiro@ie.ufrj.br
\end{abstract}


an increase in the level of external debt, which will increase the external fragility of the economy at hand.

Keywords: Endogenous growth. Foreign savings. Floating exchange rates.

JEL Classification: F43; O11; F41; E12.

\section{Introdução}

Os países desenvolvidos e em desenvolvimento têm procurado adotar políticas que acelerem o crescimento econômico, de forma a aumentar o bem-estar de seus cidadãos e reduzir a pobreza. Dentro desse contexto, diversas teorias e modelos têm sido formulados com o intuito de se identificar os fatores de crescimento das economias capitalistas. No âmbito das teorias do crescimento econômico de inspiração neoclássica, desenvolvidas a partir do trabalho seminal de Solow (1956), a poupança é vista como um elemento essencial para o crescimento de longo prazo. Nesse contexto, os países deveriam recorrer à poupança externa para aumentar a sua taxa de poupança e, dessa forma, acelerar o seu crescimento de longo prazo.

O pressuposto teórico dessa análise é que, na ausência de restrições à mobilidade internacional de capital, os capitais se moverão dos países ricos para os países pobres, ${ }^{1}$ ajudando, assim, a financiar um volume maior de investimento nos países em desenvolvimento, o que contribuiria decisivamente para a aceleração do seu crescimento. Esse processo decorre do fato de que os capitais internacionais tendem a fluir para os países que ofereçam maior remuneração (LUCAS, 1990). Sendo a remuneração do capital maior nos países em desenvolvimento, onde ele é escasso, do que nos países desenvolvidos, onde ele é abundante, os capitais se deslocariam naturalmente dos países ricos para os países pobres, contribuindo para a atenuação das desigualdades econômicas internacionais. Essa visão pró-liberalização da conta de capitais se fundamenta, portanto, na hipótese de que os capitais externos são considerados endógenos ao processo de crescimento econômico, uma vez que fluxos livres de capital promovem a eficiente alocação internacional dos recursos, com capitais fluindo em larga escala de países com elevada relação capital/trabalho para países com baixa relação capital/trabalho.

A ideia de que a poupança externa é condição necessária para o desenvolvimento econômico, no entanto, não é consenso entre os economistas. Em oposição a esses argumentos, destacam-se os trabalhos de Rodrik (1998) e Bhagwati (1998). Segundo esses autores, não há qualquer evidência de que países que se financiaram por intermédio de capitais externos melhoraram a performance de crescimento do produto e/ou tenham obtido melhorias no perfil de distribuição

1 Eliminadas as restrições à mobilidade de capitais, haveria um aumento contínuo nas transações cambiais e nos fluxos de capitais internacionais, beneficiando os países em desenvolvimento. 
de renda. Uma segunda linha de argumentação contra a tese de crescimento com poupança externa foi elaborada mais recentemente por Bresser e Nakano (2003) e Oreiro (2004). ${ }^{2}$ Para esses autores, o financiamento via poupança externa tende a gerar uma redução da taxa de crescimento de longo prazo das economias dos países em desenvolvimento devido ao aumento explosivo do endividamento externo decorrente dessa estratégia de crescimento.

Dado isso, o presente artigo irá apresentar um modelo macrodinâmico de inspiração pós-keynesiana com o objetivo de analisar os efeitos da abertura da conta de capitais do balanço de pagamentos sobre a performance macroeconômica (produto real, taxa de juros e taxa de inflação) das economias em desenvolvimento. O modelo aqui apresentado considera uma economia que possui as seguintes características: a) regime de câmbio flutuante; b) empresas oligopolistas que fixam os preços com base num mark-up fixo sobre os custos diretos unitários de produção de forma que o ajuste entre poupança e investimento é feito por intermédio de variações no grau de utilização da capacidade produtiva; e c) capitalistas domésticos que têm acesso ao mercado internacional de capitais para financiarem as suas decisões de consumo e de investimento por intermédio da emissão de títulos privados denominados em moeda estrangeira.

O modelo em consideração será usado para analisar a sensibilidade da economia frente a choques exógenos como, por exemplo, variações nas taxas de juros doméstica e internacional, aumento do endividamento externo como proporção do estoque de capital e alterações na participação dos lucros na renda. Os exercícios de estática comparativa mostram que um aumento do endividamento externo pode ter efeitos benéficos para a economia em curto prazo, uma vez que induz um aumento do valor de equilíbrio de curto prazo do grau de utilização da capacidade produtiva e da taxa de crescimento do estoque de capital.

A dinâmica de longo prazo do modelo apresentado mostra que, sob certas condições, o acesso ao mercado internacional de capital pode gerar trajetórias explosivas para o sistema econômico, notadamente num contexto em que a política monetária é conduzida com base num regime de metas de inflação. Além disso, demonstra-se que uma redução da meta de inflação perseguida pelas autoridades

2 Deve-se ressaltar que o modelo aqui apresentado possui diferenças bastante significativas com relação ao modelo de Oreiro (2004). Em primeiro lugar, no modelo de Oreiro o lado monetário da economia não está especificado, sendo um modelo que trata apenas do lado real da economia. No modelo aqui proposto iremos não só acrescentar o lado monetário, como ainda especificar uma regra de Taylor para a dinâmica da taxa nominal de juros. Em segundo lugar, o modelo de Oreiro tem uma especificação pouco usual da função investimento, ao passo que no modelo aqui apresentado a função investimento segue a especificação padrão adotada na literatura pós-keynesiana. Por fim, no modelo de Oreiro a função poupança não considera os efeitos da remessa de juros para o exterior sobre a poupança disponível dos capitalistas domésticos. Tal omissão é sanada no modelo agora em consideração, o que tem implicações bastante importantes sobre a determinação do equilíbrio de curto prazo do modelo, bem como sobre a sua dinâmica de longo prazo. 
monetárias num contexto de abertura da conta de capitais do balanço de pagamentos pode gerar um aumento permanente da taxa real de juros - com reflexos negativos sobre a acumulação de capital - bem como um aumento do endividamento externo da economia, contribuindo, assim, para uma maior fragilidade externa.

O presente artigo se divide em sete seções, incluindo a presente introdução: a seção 2 descreve a tecnologia empregada pelas firmas dessa economia; a seção 3 apresenta a fixação de preços por parte das empresas e a determinação da distribuição de renda entre salários e lucros; a seção 4 se preocupa com os determinantes da demanda efetiva; a seção 5 está dedicada à análise do equilíbrio de curto prazo do modelo; a seção 6 apresenta a dinâmica de longo prazo do modelo; e a seção 7 sumariza as conclusões obtidas ao longo do artigo.

\section{A Tecnologia}

As firmas produzem um bem homogêneo que é destinado tanto para consumo quanto para investimento. A produção de bens e serviços é feita com base numa tecnologia de produção de coeficientes fixos à la Leontieff, dada pela seguinte equação:

$$
X=\min \left[\frac{L}{b} ; K u\right]
$$

em que $L$ é a quantidade de trabalho empregada na economia; $b$ é o requisito unitário de mão de obra; $X$ é o produto real; $K$ é o estoque de capital; e $u$ é a relação produto/capital, uma proxy do grau de utilização da capacidade produtiva.

Deve-se ressaltar que os insumos capital e trabalho usados no processo produtivo são considerados homogêneos. Sendo assim, não há nenhuma diferenciação entre os diversos itens que compõem o estoque de capital da economia, tanto em nível da idade do equipamento quanto da sua qualidade. Em outras palavras, o capital é composto por itens de "safras" equivalentes. De forma análoga assumimos que o trabalho é um insumo homogêneo, não havendo nenhum tipo de distinção entre os trabalhadores no que se refere ao seu nível de qualificação. Tais hipóteses são amplamente aceitas e adotadas pela literatura pós-keynesiana sobre o tema, como, por exemplo, em Dutt (2002), Ros (2001) e Taylor (2004).

Iremos supor uma economia na qual as firmas operam com capacidade produtiva excedente de forma que toda a variação da demanda agregada é atendida por intermédio de variações do grau de utilização da capacidade 
existente. Dessa forma, a demanda de trabalho por parte das firmas será dada pela seguinte expressão:

$$
L=b X
$$

\section{Formação de Preços e Distribuição de Renda}

As firmas dessa economia operam numa estrutura de mercado oligopolista, de tal maneira que têm poder de fixação de preços. Iremos supor que as empresas fixam os preços de seus produtos com base em uma mark-up fixa sobre os custos diretos de produção. Tal como em Kalecki (1954), a distribuição da renda entre salários e lucros é determinada pela política de formação de preços das firmas, ou seja, pelas suas decisões a respeito do nível da taxa de mark-up sobre os custos diretos de produção. Supondo que o trabalho e os insumos importados são os únicos custos diretos de produção, temos que as empresas irão fixar os preços dos seus produtos com base na seguinte equação:

$$
P=(1+\tau)\left[w b+m e P^{*}\right]
$$

em que $\tau$ é o mark-up; $w$ é o salário nominal; $e$ é o câmbio nominal; $P^{*}$ é o índice de preço internacional; e $m$ é o requisito unitário de matérias-primas importadas por unidade de produto. ${ }^{3}$

Os lucros são determinados com base na diferença entre o valor nominal da produção e os custos com salários e insumos importados:

$$
P \Pi=P X-w b X-m e P^{*} X
$$

em que $\Pi$ é o montante de lucros em termos reais; $P X$ é o valor nominal da produção; $w b X$ é a folha de salários; e $m e P^{*} X$ é o valor nominal em moeda doméstica dos insumos importados.

3 É importante observar que: $m=\frac{\mathrm{M}}{X}$. 
Sendo assim, a participação dos lucros na renda é dada por: ${ }^{4}$

$$
\pi=\frac{\tau}{1+\tau}
$$

A equação 5 reproduz um dos resultados básicos da teoria de Kalecki, segundo a qual a participação dos lucros na renda é determinada, a nível microeconômico, pelo grau de monopólio das firmas (KALECKI, 1971).

A taxa de lucro é obtida ao se dividir a equação 4 por $P K$ :

$$
r=\frac{X}{P K}\left[P-w b-m e P^{*}\right]
$$

Substituindo as equações 2 e 3 na equação 6, obtém-se:

$$
r=\frac{\tau}{1+\tau} u \quad \text { ou } \quad r=\pi \cdot u
$$

Com base na equação 7, podemos observar que a taxa de lucro depende apenas da participação dos lucros na renda e do grau de utilização da capacidade produtiva, tal como ocorre em uma economia fechada e sem governo. ${ }^{5}$

Dividindo-se a equação 3 por $p$, obtém-se a seguinte expressão:

$$
V=\frac{1}{b}[(1-\pi)-q m]
$$

em que $V=\frac{W}{P}$ é o salário real; e $q=\frac{e P^{*}}{P}$ é a taxa real de câmbio.

Sendo assim, a distribuição de renda nessa economia envolve três variáveis fundamentais, a saber: o salário real, a taxa real de câmbio e a participação dos

4 A participação dos lucros na renda é definida como: $\pi=\frac{P X-w b X-m e P^{*} X}{P X}$. Temos então que $\pi=\frac{P-w b-m e P^{*}}{P}=\frac{(1+\tau)\left[w b+m e P^{*}\right]-w b-m e P^{*}}{(1+\tau)\left[w b+m e P^{*}\right]}=\frac{\tau}{(1+\tau)}$

5 Ver Taylor (1991). 
lucros na renda. A participação dos lucros na renda é determinada pela equação 5, a partir das decisões microeconômicas a respeito da fixação da taxa de mark-up. Sendo assim, temos ainda duas variáveis endógenas a serem determinadas por intermédio da equação 8: a taxa de salário real e a taxa real de câmbio, fazendo com que a distribuição de renda esteja indeterminada.

Na literatura pós-keynesiana, seguindo o trabalho seminal de Marglin (1984), consagrou-se uma metodologia de "fechos" para lidar com o problema de indeterminação do sistema. Os fechos consistem em tomar certas variáveis como parâmetros exógenos ao modelo, justificando a escolha das variáveis a se tornar parâmetros com base em argumentos de natureza institucional. Mais especificamente, os parâmetros do sistema seriam aquelas variáveis para as quais os fatores econômicos têm pouca ou nenhuma relevância para a sua determinação.

Nesse contexto, iremos adotar um fecho marxista para a equação 8 , o qual consiste em supor que a economia em consideração possui um setor de subsistência ou tradicional que funciona como uma reserva de mão de obra para as empresas que operam no setor capitalista. ${ }^{6} \mathrm{~A}$ existência desse excesso estrutural de força de trabalho faz com que, do ponto de vista do setor capitalista, a oferta de trabalho seja infinitamente elástica a um dado nível de salário real, ${ }^{7}$ o qual iremos supor como exógeno e determinado pelas convenções sociais existentes na economia num determinado momento de sua história (cf. BORTIS, 1997). Dessa forma, temos que:

$$
V=\bar{V}
$$

\section{Demanda Efetiva, Inflação e Conflito Distributivo}

A economia em consideração possui relações com o exterior e atividades governamentais. No entanto, iremos assumir que o governo não conduz nenhum tipo de atividade fiscal, de forma que o único instrumento ativo de política macroeconômica é a política monetária, sendo que esta é conduzida por intermédio de operações de mercado aberto com vistas à fixação da taxa básica de juros.

A abertura da economia abrange três noções distintas: a) a abertura do mercado de bens, entendida como a possibilidade de que as empresas e consumidores escolham entre bens nacionais e importados; b) a abertura dos mercados financeiros, entendida como a possibilidade em que os agentes domésticos escolham entre

\footnotetext{
6 Essa mesma estratégia de eliminação de indeterminação de modelos macroeconômicos é adotada por Dutt (1990).

7 A mesma hipótese foi feita por Lewis (1954).
} 
ativos financeiros nacionais e estrangeiros; c) a abertura do mercado de fatores, entendida como a possibilidade das empresas escolherem a localização de suas plantas produtivas em torno do mundo e a liberdade dos trabalhadores migrarem para outros países (BLANCHARD, 2001, p. 375-376). No modelo aqui apresentado, contudo, iremos trabalhar apenas com os dois primeiros conceitos de abertura econômica.

Seguindo Bertella e Lima (2001), iremos supor que as transações comerciais das firmas com o exterior são determinadas pelas funções de exportação e importação (normalizadas em termos do estoque de capital), dadas por:

$$
\begin{aligned}
& \frac{\mathrm{E}}{K}=\varepsilon=\varepsilon_{0}+\varepsilon_{1} q-\varepsilon_{2} u+\varepsilon_{3} u^{*} \\
& \frac{\mathrm{M}}{K}=M=M_{0}-M_{1} q+M_{2} u
\end{aligned}
$$

em que $\varepsilon_{I}$ e $M_{I}$ são parâmetros positivos; $q$ é a taxa real de câmbio; e $u{ }^{*}$ é o grau de utilização da capacidade produtiva das firmas que operam no exterior.

O saldo da balança comercial é definido como sendo a diferença entre as equações 10 e 11, de tal sorte que:

$$
(\varepsilon-M)=\left(\varepsilon_{0}-M_{0}\right)+\left(\varepsilon_{1}+M_{1}\right) q-\left(\varepsilon_{2}+M_{2}\right) u+\varepsilon_{3} u^{*}
$$

Diferenciando-se a equação 12 com respeito ao câmbio real, obtemos a seguinte expressão:

$$
\frac{\partial(\varepsilon-M)}{\partial q}=\left(\varepsilon_{1}+M_{1}\right)>0
$$

Dessa forma, observa-se que uma desvalorização do câmbio real, dado o grau de utilização da capacidade produtiva, produz um inequívoco aumento do saldo da balança comercial, mostrando, assim, a validade da condição de Marshall-Lerner. $^{8}$

Substituindo a equação 9 na 8, chegamos a seguinte expressão:

8 Sobre a condição de Marshall-Lerner, ver Argy (1994, p. 144) e Carlin e Soskice (2006, p. 341). 


$$
q=\frac{1}{m}[(1-\pi)-\overline{V b}]
$$

Dividindo-se a equação 11 por $\boldsymbol{u}$ e substituindo a resultante na 13, obtemos após os algebrismos necessários, a seguinte equação:

$$
u=\frac{M_{1} q^{2}-M_{0} q}{M_{2} q-(1-\pi)+\bar{V} b}
$$

A equação 15 descreve o lócus LD, definido como sendo o conjunto das combinações entre o grau de utilização da capacidade produtiva e taxa real de câmbio $(u, q)$ para as quais há equilíbrio distributivo na economia. Isto é, uma situação na qual o mark-up efetivo é igual ao desejado, o salário real efetivo é igual ao nível determinado pelas convenções sociais prevalecentes na economia e a taxa real de câmbio é aquela permitida pela equação 8.

Diferenciando a equação 14 com respeito à $u$ e $q$, obtém-se a expressão 15, que apresenta a inclinação do lócus LD:

$$
\frac{\partial u}{\partial q} \mid L D=\frac{\left(M_{0}-2 M_{1} q\right)(1-\pi-\bar{V} b)+M_{1} M_{0} q^{2}}{\left[M_{2} q-(1-\pi-\bar{V} b)\right]^{2}}
$$

Com base em na expressão 15, constatamos que a inclinação do lócus LD depende do valor assumido pela taxa real de câmbio. Com efeito, se $q>\frac{M_{0}}{2 M_{1}} \Rightarrow \frac{\partial u}{\partial q}<0$, caso contrário temos que $\frac{\partial u}{\partial q}>0$.

Seguindo Robinson (1962) e Taylor (1985), iremos supor que a taxa desejada de crescimento do estoque de capital por parte das firmas depende de dois componentes: um componente autônomo que capta o "otimismo espontâneo" dos capitalistas, ou seja, o seu animal spirits, e outro componente que depende da diferença entre a taxa de retorno do capital e a taxa real de juros. Dessa forma, podemos escrever a seguinte equação:

$$
\frac{I}{K}=g^{i}=g_{0}+h[r-i-\hat{P}]
$$


em que $I$ é o investimento agregado; $g^{i}$ é a taxa desejada de crescimento do estoque de capital; $g_{0}$ representa o animal spirits dos capitalistas; $h$ mede a sensibilidade da taxa desejada de crescimento do estoque de capital às diferenças entre a taxa de lucro e a taxa real de juros; i é a taxa nominal de juros; e $\hat{P}$ é a taxa corrente de inflação.

Tal como Rowthorn (1977), iremos supor que a inflação advém do conflito distributivo entre o capital e o trabalho. A inflação salarial irá ocorrer toda vez que os trabalhadores desejarem uma participação dos salários (dos lucros) na renda nacional maior (menor) do que o valor corrente dessa variável. Dessa forma, podemos representar a inflação salarial por intermédio de:

$$
\hat{w}=\psi\left[\pi-\pi^{F}\right]
$$

em que $w$ é a taxa proporcional de variação do salário nominal; $\psi$ indica a velocidade de ajustamento, sendo que $0<\psi<1$; e $\pi^{F}$ é a participação nos lucros na renda desejada pelos trabalhadores.

A participação dos lucros na renda desejada pelos trabalhadores depende claramente do poder de barganha destes no mercado de trabalho. Dessa forma, iremos supor que o aumento do nível de emprego aumenta o poder de barganha dos trabalhadores e, portanto, a parcela da renda de que desejam se apropriar. Como, pela equação 2, o emprego depende do nível de produção, segue-se que pode-se utilizar o grau de utilização da capacidade produtiva como uma proxy para o nível de emprego. Sendo assim, tem-se que:

$$
\pi^{F}=\alpha_{0}-\alpha_{1} u
$$

Como o salário real é por hipótese constante, segue-se que a inflação salarial é instantaneamente repassada para os preços. Sendo assim, tem-se que:

$$
\hat{P}=\hat{w}
$$

Substituindo a equação 18 na 17, e a equação resultante na 19, obtém-se a seguinte: 


$$
\hat{P}=\psi \pi-\psi \alpha_{0}+\psi \alpha_{1} u
$$

A equação 20 é uma espécie de curva de Phillips para a economia em consideração na medida em que estabelece uma relação inversa entre a taxa de inflação e o grau de utilização da capacidade produtiva.

Substituindo a equação 20 na 16, obtém-se a seguinte expressão para a taxa desejada de crescimento do estoque de capital:

$$
g^{i}=g_{0}-h \psi \alpha_{0}+h\left[\pi-\psi \alpha_{1}\right] u-h i-h \psi \pi
$$

Seguindo a tradição de Marx (1988), Kalecki (1971, 1954), Kaldor (1957, 1956), Robinson $(1962,1956)$ e Pasinetti (1962), iremos supor que os capitalistas recebem sob a forma de lucros todo o excedente sobre os salários, poupando uma fração constante de sua renda $\left(s_{\pi}\right)$. Já os trabalhadores gastam toda a sua renda com consumo.

Deve-se ressaltar, contudo, que uma parte da renda dos capitalistas domésticos não está disponível para o financiamento dos seus gastos de consumo, pois é remetida para o exterior na forma de encargos financeiros sobre a dívida externa do setor privado. Dessa forma, a poupança dos capitalistas pode ser apresentada pela seguinte equação:

$$
P S=s_{\pi}\left(r P K-e i^{*} B\right)=s_{\pi}\left(r P K-e i^{*} Z K\right)
$$

em que $S$ é a poupança dos capitalistas; $s_{\pi}$ é a propensão a poupar a partir dos lucros; $i^{*}$ é a taxa de juros dos títulos internacionais; $B$ é o estoque da dívida externa do setor privado; e $Z$ é o endividamento externo como proporção do estoque de capital.

No que se refere ao financiamento externo, iremos assumir que apenas as firmas que possuam um valor mínimo de capital e lucros estão dispostas e capazes de demandar poupança externa para complementar a sua própria acumulação interna de capital. Esse financiamento é estritamente privado e realizado por intermédio de emissão de títulos à taxa de juros $\left(i^{*}\right)$.

Nesse contexto, é importante destacar que essa economia opera num regime de mobilidade imperfeita de capitais com câmbio flutuante, no sentido de Mundell (1962) e Fleming (1962), de tal forma que a existência de ganhos de arbitragem entre títulos domésticos e os títulos internacionais dá origem a um fluxo de entra- 
da/saída de capitais finito por unidade de tempo. Isso porque estamos supondo que os títulos doméstico e internacional são substitutos imperfeitos entre si. Dessa forma, não vale a equação de paridade descoberta da taxa de juros. Além disso, a hipótese de pequena economia aberta assegura que a taxa de juros doméstica não tem efeito sobre a taxa de juros internacional.

Dividindo-se a equação 22 por $P K$, e após alguns algebrismos, ${ }^{9}$ encontra-se:

$$
g^{S}=s_{\pi} \pi u-\frac{s_{\pi} i^{*} Z}{P^{*}} q
$$

em que $g^{S}$ é a taxa de crescimento do estoque de capital permitida pela disponibilidade de poupança dessa economia.

Em curto prazo, admite-se que o estoque de capital $(K)$, o coeficiente unitário de mão de obra $(b)$ e o mark-up $(\tau)$ são constantes. Dessa forma, o grau de utilização da capacidade produtiva é a variável de ajuste entre a taxa de crescimento do estoque de capital que é desejada pelas firmas e a taxa de crescimento do estoque de capital que é permitida pela disponibilidade de poupança da economia. A condição de equilíbrio macroeconômico é dada então por:

$$
g^{i}=g^{S}-(\varepsilon-M)
$$

Substituindo as equações 10, 11, 21 e 23 na equação 24), e resolvendo para $u$, obtém-se após os necessários algebrismos a seguintes expressão:

$$
u=\frac{1}{S_{\pi} \pi-h\left[\pi-\psi \alpha_{1}\right]+\varepsilon_{2}+M_{2}}\left[\left(g_{0}+h \psi \alpha_{0}+\varepsilon_{0}-M_{0}\right)-h i-h \psi \pi+\left(\frac{s_{\pi}{ }^{*} Z}{P^{*}}+\varepsilon_{1}+M_{1}\right) q\right]
$$

A equação 25 descreve o lócus IS, definido como sendo o conjunto das combinações entre grau de utilização da capacidade produtiva e câmbio real (u, q), para os quais o mercado de bens está em equilíbrio.

Para obter a inclinação do lócus IS basta diferenciar a equação 25 com respeito a $u$ e a $q$, de forma a se obter a seguinte expressão:

Sabemos que $\frac{P S}{P K}=g^{S}=s_{\pi} r-\frac{s_{\pi} e i^{*} Z}{P}=s_{\pi} \pi u-\frac{s_{\pi} e i^{*} Z P^{*}}{P P^{*}}=s_{\pi} \pi u-\frac{s_{\pi} i^{*} Z}{P^{*}} q$, sendo que
$r=\pi u$. 


$$
\frac{\partial u}{\partial q} \mid I S=\frac{1}{s_{\pi} \pi-h\left[\pi-\psi \alpha_{1}\right]+\varepsilon_{2}+M_{2}}\left[\frac{s_{\pi} i^{*} Z}{P^{*}}+\varepsilon_{1}+M_{1}\right]>0
$$

Uma depreciação da taxa real de câmbio gera um aumento do grau de utilização da capacidade produtiva. Isso decorre tanto do efeito positivo que a desvalorização da taxa real de câmbio tem sobre as exportações líquidas (dado que estamos supondo a validade da condição Marshall-Lerner), quanto também do fato que uma desvalorização da taxa real de câmbio ao aumentar a renda enviada para o exterior (devido aos encargos financeiros da dívida externa privada) reduz a poupança dos capitalistas domésticos. Dessa forma, o ajuste no mercado de bens exige um aumento do grau de utilização da capacidade produtiva. ${ }^{10}$

Diferenciando a equação 25 , com respeito a Z, obtém-se:

$$
\frac{\partial u}{\partial Z}=\frac{1}{s_{\pi} \pi-h\left[\pi-\psi \alpha_{1}\right]+\varepsilon_{2}+M_{2}}\left(\frac{s_{\pi} i^{*}}{P^{*}}\right)>0
$$

Um aumento do endividamento externo (como proporção do estoque de capital) irá aumentar o grau de utilização da capacidade produtiva. Isso porque um aumento do endividamento externo reduz a renda disponível dos capitalistas (pós-pagamento dos encargos da dívida externa privada), levando-os a reduzir a sua poupança. Dessa forma, o ajuste no mercado de bens também termina por exigir um aumento do nível de utilização da capacidade produtiva.

\section{A Configuração de Equilibrio de Curto Prazo e a Estática Comparativa}

A economia estará em equilíbrio de curto prazo quando o grau de utilização da capacidade produtiva $(u)$ e a taxa real de câmbio forem tais a satisfazer simultaneamente a equação 14 - referente ao lócus LD - e a equação 25 - referente ao lócus IS. Deve-se enfatizar que o curto prazo é definido como aquele intervalo de tempo para o qual o endividamento externo como proporção do estoque de capital e a taxa doméstica de juros são mantidas constantes. Em longo prazo, con-

10 A literatura de macroeconomia aberta tem apontado para a possibilidade de que uma desvalorização da taxa de câmbio nominal possa produzir um efeito contracionista sobre o nível de equilíbrio de emprego (cf. AGÉNOR; MONTIEL, 1999, p. 286). No entanto, os modelos formais mais recentes sobre tal efeito, como, por exemplo, Agénor e Montiel (1999), assumem a existência de um regime de câmbio fixo no contexto de um modelo de economia dependente no qual existe mobilidade do fator de produção trabalho a nível internacional. A diferença entre os resultados do modelo aqui apresentado e o modelo de economia dependente de Agénor e Montiel (1999) se fundamenta no fato de que no primeiro modelo assume-se a existência de um regime de câmbio flutuante, ao passo que no segundo modelo é assumida a existência de um regime de câmbio fixo. 
tudo, a dívida externa e a taxa de juros são variáveis endógenas. A endogenização dessas variáveis será feita na próxima seção.

Como a inclinação do lócus LD muda quando a taxa real de câmbio ultrapassa certo valor crítico dado por $q_{c}=\frac{M_{0}}{2 M_{1}}$, existem pelo menos duas configurações possíveis para o equilíbrio de curto prazo, tal como pode ser observado na Figura 1.

Figura 1 - Possíveis configurações de equilíbrio de curto prazo
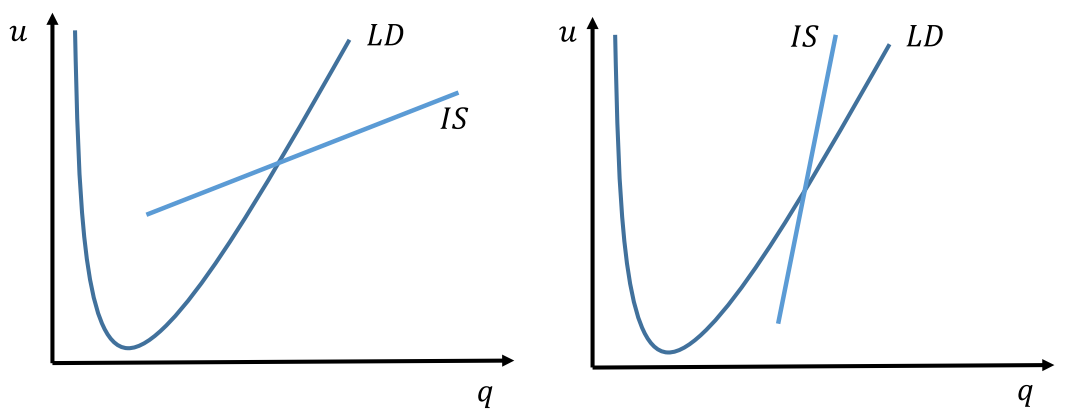

Fonte: Elaboração própria.

Pode-se demonstrar que apenas a situação representada pelo caso A refere-se a um equilíbrio de curto prazo estável. Dessa forma iremos supor que $\frac{\partial u}{\partial q}\left|L D>\frac{\partial u}{\partial q}\right| L S$. Para encontrar a taxa real de câmbio de equilíbrio de curto prazo do sistema, iguala-se a equação 14 com a equação 25. Dessa forma, obtém-se a seguinte expressão:

$$
\begin{aligned}
& \frac{M_{1} q^{2}-M_{0} q}{M_{2} q-(1-\pi-\bar{V} b)}-\frac{1}{S_{\pi} \pi-h\left[\pi-\psi \alpha_{1}\right]+\varepsilon_{2}+M_{2}}\left[\left(g_{0}+h \psi \alpha_{0}+\varepsilon_{0}-M_{0}\right)-h i-h \psi \pi\right. \\
& \left.+\left(\frac{s_{\pi}{ }^{*} Z}{P^{*}}+\varepsilon_{1}+M_{1}\right) q+\varepsilon_{3}\right]=0
\end{aligned}
$$

Iremos utilizar a expressão 27 para calcular os efeitos de mudanças exógenas da taxa de juros internacional, da taxa de juros doméstica, da dívida externa como proporção do estoque de capital e da participação dos lucros na renda sobre o valor de equilíbrio de curto prazo da taxa real de câmbio. Diferenciando a equação 27 com respeito a $i, i^{*}, Z$ e $\pi$, obtém-se: 


$$
\frac{\partial q}{\partial i}=-\frac{\varphi_{2}}{\varphi_{1}}<0
$$

$$
\frac{\partial q}{\partial Z}=-\frac{\varphi_{3}}{\varphi_{1}}>0
$$

$$
\frac{\partial q}{\partial i^{*}}=-\frac{\varphi_{4}}{\varphi_{1}}>0
$$

$$
\frac{\partial q}{\partial \pi}=-\frac{\varphi_{5}}{\varphi_{1}}=?
$$

em que

$$
\begin{aligned}
& \frac{\left(M_{0}-2 M_{1} q\right)(1-\pi-\bar{V} b)+M_{1} M_{0} q^{2}}{\left[M_{2} q-(1-\pi-\bar{V} b)\right]^{2}}-\frac{1}{s_{\pi} \pi-h\left[\pi-\psi \alpha_{1}\right]+\varepsilon_{2}+M_{2}}\left[\frac{s_{\pi^{*}}{ }^{*} Z}{P^{*}}+\varepsilon_{1}+M_{1}\right]=\varphi_{1}>0 \\
& \frac{h}{s_{\pi} \pi-h\left[\pi-\psi \alpha_{1}\right]+\varepsilon_{2}+M_{2}}=\varphi_{2}>0 \\
& -\frac{s_{\pi} q i^{*}}{\left[s_{\pi} \pi-h\left[\pi-\psi \alpha_{1}\right]+\varepsilon_{2}+M_{2}\right] P^{*}}=\varphi_{3}<0 \\
& -\frac{s_{\pi} q Z}{\left[s_{\pi} \pi-h\left[\pi-\psi \alpha_{1}\right]+\varepsilon_{2}+M_{2}\right] P^{*}}=\varphi_{4}<0 \\
& \frac{g_{0}+h \psi \alpha_{0}+\varepsilon_{0}-M_{0}-h[i+\psi \pi]+\left[\frac{s_{\pi} i^{*} Z}{P^{*}}+\varepsilon_{1}+M_{1}\right] q+u^{*}}{\left[s_{\pi} \pi-h\left[\pi-\psi \alpha_{1}\right]+\varepsilon_{2}+M_{2}\right]^{2}}\left(s_{\pi}-h\right)+\frac{h \psi}{s_{\pi} \pi-h\left[\pi-\psi \alpha_{1}\right]+\varepsilon_{2}+M_{2}}=\varphi_{5}
\end{aligned}
$$

A expressão 28a aponta que um aumento da taxa de juros doméstica gera uma apreciação do cambio real. Com efeito, um aumento da taxa de juros doméstica irá reduzir a taxa de crescimento do estoque de capital que é desejada pelas firmas, deslocando para baixo a curva IS. Dada a distribuição de renda, tem-se um aumento do requisito de matéria-prima importada por unidade de produto, de tal forma que a taxa real de câmbio se aprecia.

A expressão 28b nos mostra que um aumento do endividamento externo como proporção do estoque de capital gera uma depreciação da taxa de câmbio real de equilíbrio, pois um aumento do endividamento externo irá resultar num aumento da renda que os capitalistas domésticos têm que remeter para o exterior na forma de encargos financeiros (juros + amortizações). Dessa forma, ocorre uma 
redução da poupança dos capitalistas e, portanto, um aumento dos seus gastos de consumo. Esse aumento do consumo desloca a curva IS para cima de tal forma que o grau de utilização da capacidade produtiva aumenta. Dada a distribuição de renda entre salários e lucros, o requisito de matéria-prima importada por unidade de produto se reduz, depreciando, portanto, o câmbio real.

Dada a ambiguidade no coeficiente de $\varphi_{5}$, algumas considerações se fazem necessárias. Em curto prazo, o efeito do aumento/redução dos lucros sobre o câmbio real dependerá da elasticidade das exportações e importações com respeito à taxa de câmbio real, ${ }^{11}$ porque, dada a validade da condição de Marshall-Lerner, a resposta da balança comercial em relação a mudanças na distribuição de renda é lenta de tal forma que, por exemplo, a elevação dos lucros na renda aumenta o consumo de produtos importados, o que se traduz em uma piora da balança comercial.

Por outro lado, essa mudança na balança comercial pode se traduzir em uma redução do investimento privado em curto prazo em razão de perda de competitividade externa, o que reduziria o grau de utilização da capacidade produtiva. Acrescenta-se que o aumento da participação dos lucros na renda reduz a participação relativa dos salários no produto, que deve cair ainda mais com a depreciação da moeda, o que aumenta a queda da demanda e do produto. De fato, para uma economia aberta, como a considerada nesse modelo, uma depreciação da moeda é equivalente a uma redução dos salários (cf. LÓPEZ, 2001, p. 297).

No geral, o que a experiência histórica tem mostrado é que a depreciação da moeda possui influências negativas sobre a demanda e o produto. ${ }^{12}$ É importante salientar nesta análise que os segmentos mais pobres da população sofrem mais com a queda no produto e no emprego. No entanto, o declínio econômico provocado pela elevação dos lucros na renda também afeta o padrão de vida dos segmentos de renda mais elevada, dada a queda do produto. Como resultado final desta discussão, admite-se que $\frac{\partial q}{\partial \pi}<0$.

Os efeitos de variações da taxa doméstica de juros, da taxa de juros internacional, da dívida externa como proporção do estoque de capital e da participação dos lucros na renda podem ser analisados por intermédio das seguintes derivadas parciais: ${ }^{13}$

11 Se as firmas exportadoras não estiverem dispostas a reduzir seus preços no mercado internacional, dado o seu poder de monopólio, então a demanda por exportações não será afetada de forma substancial e o efeito final da balança comercial dependerá da elasticidade-preço das exportações.

12 Ver Taylor (1998).

13 Essas expressões podem ser obtidas ao se substituir e equação 27 na 14 e se diferenciar a expressão resultante com respeito a $i, i^{*}, Z$ e $\pi$. 


$$
\begin{gathered}
\frac{\partial u}{\partial i}=-\frac{\theta_{2}}{\theta_{1}}<0 \\
\frac{\partial u}{\partial Z}=-\frac{\theta_{3}}{\theta_{1}}>0 \\
\frac{\partial u}{\partial i^{*}}=-\frac{\theta_{4}}{\theta_{1}}>0 \\
\frac{\partial u}{\partial \pi}=-\frac{\theta_{5}}{\theta_{1}}<0
\end{gathered}
$$

em que:

$$
\begin{aligned}
& \frac{s_{\pi} i^{*}\left[u\left[s_{\pi} \pi-h\left[\pi-\psi \alpha_{1}\right]+\varepsilon_{2}+M_{2}\right]-\left(g_{0}+h \psi \alpha_{0}+\varepsilon_{0}-M_{0}\right)+h i+h \psi \pi-u^{*}\right]}{P^{*}\left[\frac{s_{\pi} i^{*} Z}{P^{*}}+\varepsilon_{1}+M_{1}\right]^{2}}=\theta_{3}>0 \\
& \frac{s_{\pi} Z\left[u\left[s_{\pi} \pi-h\left[\pi-\psi \alpha_{1}\right]+\varepsilon_{2}+M_{2}\right]-\left(g_{0}+h \psi \alpha_{0}+\varepsilon_{0}-M_{0}\right)+h i+h \psi \pi-u^{*}\right]}{P^{*}\left[\frac{s_{\pi} i^{*} Z}{P^{*}}+\varepsilon_{1}+M_{1}\right]^{2}}=\theta_{4}<0 \\
& -\frac{u\left(s_{\pi}-h\right)+h \psi}{\left[\frac{s_{\pi}{ }^{*} Z}{P^{*}}+\varepsilon_{1}+M_{1}\right]}=\theta_{5}<0
\end{aligned}
$$

A expressão 29a mostra que um aumento da taxa básica de juros provoca uma redução do grau de utilização da capacidade produtiva de equilíbrio de curto prazo em razão da redução da taxa desejada de crescimento do estoque de capital.

A expressão 29b mostra que um aumento da dívida externa (como proporção do estoque de capital) gera um aumento do grau de utilização da capacidade produtiva compatível com o equilíbrio de curto prazo do sistema econômico. Esse resultado é uma decorrência elementar do fato de que um aumento da dívida externa do setor privado gera uma expansão da demanda efetiva, tal como podemos observar na equação 26b. Isso equivale a um deslocamento para cima da curva IS na Figura A. O lócus LD, por sua vez, não se altera com mudanças no endividamento externo como proporção do estoque de capital. Sendo assim, haverá um 
novo equilíbrio de curto prazo com um nível de utilização da capacidade produtiva mais alto (e uma taxa de câmbio mais depreciada).

A equação $29 \mathrm{c}$ mostra que um aumento da taxa de juros internacional gera um aumento do grau de utilização da capacidade produtiva. Esse resultado pouco comum é fruto da hipótese aqui adotada a respeito do regime de mobilidade de capitais. Como a mobilidade de capitais é imperfeita no sentido de Mundell e Fleming, segue-se que um aumento da taxa de juros internacional não induz um aumento da taxa de juros doméstica. No entanto, o aumento da renda enviada para o exterior induz uma depreciação da taxa real de câmbio (ver equação 28c), o que aumenta as exportações líquidas, induzindo, dessa forma, uma expansão da demanda efetiva e do grau de utilização da capacidade produtiva de equilíbrio de curto prazo.

Por fim, a expressão $29 \mathrm{~d}$ mostra que um aumento da participação dos lucros na renda reduz o grau de utilização da capacidade produtiva.

Com base na equação 21, sabemos que $\frac{\partial g^{I}}{\partial u}>0$. Dessa forma, pela regra da cadeia, é possível afirmar que:

$$
\begin{aligned}
& \frac{\partial g^{i}}{\partial i}=\frac{\partial g^{i}}{\partial u} \frac{\partial u}{\partial i}=(+)(-)<0 \\
& \frac{\partial g^{i}}{\partial Z}=\frac{\partial g^{i}}{\partial u} \frac{\partial u}{\partial Z}=(+)(+)>0 \\
& \frac{\partial g^{i}}{\partial i^{*}}=\frac{\partial g^{i}}{\partial u} \frac{\partial u}{\partial i^{*}}=(+)(+)>0 \\
& \frac{\partial g^{i}}{\partial \pi}=\frac{\partial g^{i}}{\partial u} \frac{\partial u}{\partial \pi}=(+)(-)<0
\end{aligned}
$$

A taxa de crescimento do estoque de capital é uma função inversa da taxa nominal de juros doméstica e da participação dos lucros na renda. Dessa forma, podemos afirmar que nessa economia prevalece um regime de crescimento do tipo wage-led. Além disso, podemos afirmar que a taxa de crescimento do estoque de capital é uma função direta do endividamento externo como proporção do estoque de capital e da taxa de juros internacional.

Da mesma forma, sabe-se da equação 20 que $\frac{\partial P}{\partial u}>0$. Logo, utilizando o mesmo expediente, tem-se que: 


$$
\begin{aligned}
& \frac{\partial \hat{P}}{\partial i}=\frac{\partial \hat{P}}{\partial u} \frac{\partial u}{\partial i}=(+)(-)<0 \\
& \frac{\partial \hat{P}}{\partial Z}=\frac{\partial \hat{P}}{\partial u} \frac{\partial u}{\partial Z}=(+)(+)>0 \\
& \frac{\partial \hat{P}}{\partial i^{*}}=\frac{\partial \hat{P}}{\partial u} \frac{\partial u}{\partial i^{*}}=(+)(+)>0 \\
& \frac{\partial \hat{P}}{\partial \pi}=\frac{\partial \hat{P}}{\partial u} \frac{\partial u}{\partial \pi}=(+)(-)<0
\end{aligned}
$$

Pelas expressões 29a, 30a e 31a percebemos que a política monetária é capaz de influenciar a performance de variáveis econômicas, como inflação, grau de utilização da capacidade produtiva e taxa de crescimento do estoque de capital no contexto de uma economia aberta com mobilidade imperfeita de capitais. No entanto, se a autoridade monetária resolver reduzir a taxa de inflação, então esse objetivo só poderá ser alcançado por intermédio de uma redução da taxa de crescimento. Dentro desse contexto, percebe-se claramente que os objetivos, em curto prazo, de crescimento e baixa inflação são conflitantes.

A expressão $30 \mathrm{~b}$ mostra que, em curto prazo, o acesso ao mercado internacional de capitais e o endividamento externo a ele correlato contribuem para aumentar a taxa de crescimento do estoque de capital.

\section{A Dinâmica de Longo Prazo}

Para analisar a dinâmica de longo prazo dessa economia, é necessário explicitar as equações de movimento do modelo. Seguindo Simonsen e Cysne (1995), a equação diferencial que descreve a dinâmica temporal da dívida externa é dada por:

$$
\dot{Z}=i^{*} D-H
$$

em que $D$ é o estoque de dívida externa; e $H$ é a transferência líquida de recursos para o exterior.

Diferenciando $z$ com respeito ao tempo, obtém-se a seguinte expressão: 


$$
\dot{Z}=\frac{\dot{D}}{H}-\frac{D}{K} g^{i}
$$

Substituindo a equação 32 na 33, encontra-se:

$$
\dot{Z}=\left(i^{*}-g^{i}\right) Z-\frac{H}{K}
$$

Substituindo a equação 26 na 34, obtém-se a equação que descreve a dinâmica temporal da dívida externa como proporção do estoque de capital:

$$
\dot{Z}=Z\left(h \psi \alpha_{0}-g_{0}\right)+i^{*} Z+h i Z+h \psi \pi Z-h Z\left(\pi-\psi \alpha_{1}\right) u(i, Z, \pi)-\frac{H}{K}
$$

Iremos supor que na economia em consideração a política monetária é conduzida no contexto de um regime de metas de inflação no qual a autoridade monetária deve utilizar a taxa nominal de juros com o objetivo de obter uma meta numérica definida para a taxa de inflação em médio prazo. Dessa forma, iremos supor que a política monetária é conduzida com base numa função de reação do Banco Central dada por:

$$
i=\phi\left[\hat{P}(i, Z, \pi)-\hat{P}^{*}\right]
$$

em que $\phi$ é um parâmetro positivo que representa a velocidade com a qual o Banco Central reage a desvios entre a taxa de inflação efetiva e a meta inflacionária;

e $P^{*}$ é a meta de inflação perseguida pela autoridade monetária.

As expressões 35 e 36 formam um sistema de equações diferenciais que determinam a dinâmica da dívida externa como proporção do estoque de capital e da taxa básica de juros da economia. A matriz jacobiana do sistema é dada por: 


$$
\left[\begin{array}{c}
\dot{Z} \\
\dot{i}
\end{array}\right]=\left[\begin{array}{cc}
{\left[\left(h \psi a_{0}-g_{0}\right)+h i+i^{*}+h \psi \pi\right]-h\left(\pi-\psi a_{1}\right)\left(u+Z \frac{\partial u}{\partial Z}\right)} & h Z\left[1-\left(\pi-\psi a_{1}\right) \frac{\partial u}{\partial i}\right]
\end{array}\right]\left[\begin{array}{c}
Z-Z_{0} \\
i-i_{0}
\end{array}\right]
$$

Logo, define-se:

$$
\begin{gathered}
\lambda_{0}=\left[\left(h \psi a_{0}-g_{0}\right)+h i+i^{*}+h \psi \pi\right]-h\left(\pi-\psi a_{1}\right)\left(u+Z \frac{\partial u}{\partial Z}\right)=? \\
\lambda_{1}=h Z\left[1-\left(\pi-\psi a_{1}\right) \frac{\partial u}{\partial i}\right]>0 ; \lambda_{2}=\phi \frac{\partial \hat{P}}{\partial Z}>0 ; \quad \lambda_{3}=\phi \frac{\partial \hat{P}}{\partial i}<0
\end{gathered}
$$

Analisando o sinal dos elementos da matriz jacobiana, a única ambiguidade refere-se ao sinal de $\lambda_{0}$, que pode ser positivo ou negativo.

$$
\text { Define-se } \frac{Z}{u} \frac{\partial u}{\partial Z}=\varepsilon_{Z}>0 \text { como sendo a elasticidade do grau de utilização }
$$
da capacidade produtiva com respeito ao endividamento externo, uma medida da sensibilidade do nível de atividade econômica com respeito a variações do nível de endividamento externo do setor privado. Sendo assim, temos que:

$$
\lambda_{0}=\left[\left(h \psi a_{0}-g_{0}\right)+h i+i^{*}+h \psi \pi\right]-h\left(\pi-\psi a_{1}\right) u\left(1+\varepsilon_{z}\right)
$$

Na expressão acima observamos que o sinal de $\lambda_{0}$ depende do valor assumido por essa elasticidade. Em particular, se $\varepsilon_{z}<\frac{h \psi a_{0}-g_{0}+i^{*}+h \psi \pi}{h\left(\pi-\psi a_{1}\right) u}-1$, então $\lambda_{0}$ será positivo. No que se segue, portanto, supor-se-á que a elasticidade do grau de utilização da capacidade produtiva com respeito ao endividamento externo é baixa.

Dessa forma, o determinante e o traço da matriz jacobiana são dados por:

$$
\begin{aligned}
& \operatorname{Tr}(J)=\lambda_{0}+\lambda_{3}<0 \\
& \operatorname{Det}(J)=\left(\lambda_{0} \lambda_{3}\right)-\left(\lambda_{1} \lambda_{2}\right)<0
\end{aligned}
$$


Como o determinante da matriz jacobiana é negativo, podemos afirmar que o equilíbrio de longo prazo do sistema econômico é instável do tipo trajetória de sela (cf. TAKAYAMA, 1993, p. 407-408). Esse resultado aponta para a ideia de que a abertura da conta de capitais do balanço de pagamentos, embora possa ter efeitos benéficos para a economia em curto prazo, pode ser fonte de instabilidade a longo prazo em consonância com a argumentação desenvolvida por Bresser e Nakano (2003).

Em steady-state, o endividamento externo e a taxa básica de juros são constantes ao longo do tempo. Isso permite com que o lócus $\dot{Z}=0$ e o lócus $\dot{i}=0$, cujas inclinações são dadas respectivamente pelas seguintes equações, sejam determinados assim:

$$
\begin{aligned}
& \left.\frac{\partial i}{\partial Z}\right|_{\dot{Z}=0}>0 \\
& \left.\frac{\partial i}{\partial Z}\right|_{i=0}<0
\end{aligned}
$$

Sendo assim, a configuração de equilíbrio de longo prazo da economia em consideração pode ser visualizada por meio da Figura 2.

Figura 2 - Configuração de equilíbrio de longo prazo

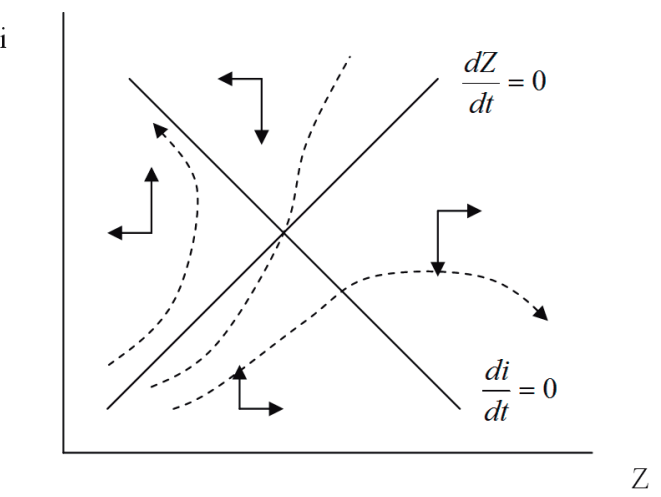

Fonte: Elaboração própria.

No que se segue, supor-se-á que a autoridade monetária ajusta a taxa nominal de juros de maneira a garantir que a economia esteja sempre sobre a sua trajetória de sela. 
Nesse contexto, suponha-se que as autoridades monetárias resolvam perseguir uma meta de inflação mais baixa. Qual seria o impacto sobre o equilíbrio de longo prazo do sistema econômico? Uma redução da meta de inflação irá deslocar o lócus $\frac{d i}{d t}=0$ para cima e para a direita, determinando um novo equilíbrio de longo prazo no qual a taxa nominal de juros e o endividamento externo como proporção do estoque de capital são ambos mais elevados (Figura 3). Como no equilíbrio de longo prazo a taxa de inflação efetiva deve ser igual à meta inflacionária, segue-se que a taxa efetiva de inflação irá se reduzir, impondo, assim, um aumento da taxa real de juros. Daqui se segue, portanto, que uma redução da meta de inflação resulta num aumento da taxa real de juros e do endividamento externo (como proporção do estoque de capital) de equilíbrio de longo prazo do sistema econômico.

Figura 3 - Efeitos de longo prazo de uma redução da meta de inflação

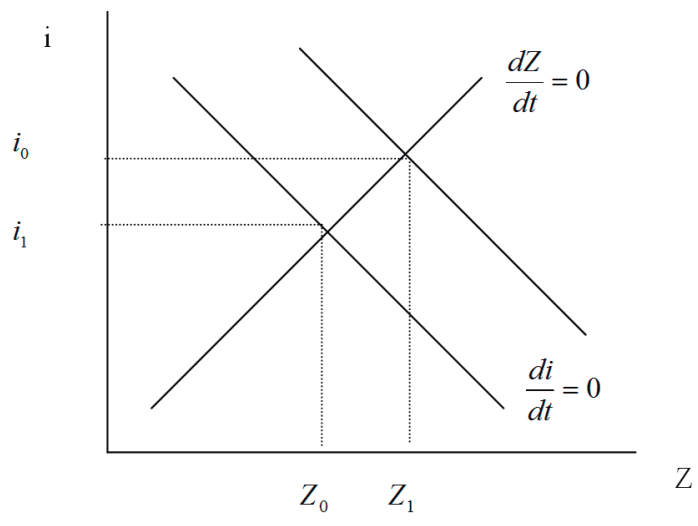

Fonte: Elaboração própria.

\section{Considerações Finais}

Ao longo do presente artigo foi desenvolvido um modelo dinâmico não linear de inspiração pós-keynesiana para avaliar a performance, em curto e em longo prazo, de uma série de variáveis macroeconômicas num contexto de mobilidade (limitada) de movimentação de capitais. O modelo aqui apresentado mostra que em curto prazo um aumento do endividamento externo tem impacto positivo sobre a performance macroeconômica. Mais especificamente, um aumento do endividamento externo gera um aumento do valor de equilíbrio de curto prazo do grau de utilização da capacidade produtiva e da taxa de crescimento do estoque de capital. Contudo, em longo prazo, a abertura da conta de capitais do balanço de pagamentos pode ter efeitos desestabilizadores sobre o sistema econômico. 
Além disso, a análise de longo prazo do sistema econômico mostrou que uma redução da meta de inflação a ser perseguida pela autoridade monetária produz uma elevação da taxa real de juros e do endividamento externo de equilíbrio de longo prazo. Dessa forma, uma política monetária contracionista estará associada a um aumento permanente do custo de oportunidade do capital (podendo ter um efeito negativo sobre o crescimento de longo prazo) e um aumento da fragilidade externa da economia.

\section{Referências}

AGÉNOR, P-R.; MONTIEL, P. Development Macroeconomics. Princeton University Press: Princeton, 1999.

ARGY, V. International Macroeconomics. Routledge: Londres. 1994.

BERTELLA, M. A.; LIMA, G. T. Termos de troca, salário real e nível de atividade. Revista da Sociedade Brasileira de Economia Política, São Paulo, v. 9, n. 9, p. 86-106, 2001.

BHAGWATI, J. Yes to free trade, maybe to capital controls. Wall Street Journal, p. A38, Nov. 16, 1998.

BLANCHARD, O. Macroeconomia. Campus: Rio de Janeiro, 2001.

BORTIS, H. Institutions, behaviour and economic theory: a contribution to classical-Keynesian political economy. Cambridge: Cambridge University Press, 1997.

BRESSER, L. C.; NAKANO, Y. Crescimento econômico com poupança externa? Revista de Economia Política, v. 23, n. 2, p. 3-27, 2003.

CALVO, G. Crises de Balanços de Pagamentos em Mercados Emergentes. In: KRUGMAN, P. (Org.). Crises monetárias. São Paulo: Makron Books, 2001.

CARLIN, W.; SOSKICE, D. Macroeconomics: imperfections, institutions, and policies. Oxford: Nova Iorque, 1996.

DUTT, A. K. Growth, distribution and uneven development. Cambridge: Cambridge University Press, 1990.

. Thirlwall's law and uneven development. Journal of Post Keynesian Economics, v. 23, n. 3, p. 367-390, Spring, 2002.

FLEMING, J. M. Domestic financial policies under fixed and under flexible exchange rates. Washington, D.C.: IMF, 1962. (International Monetary Fund Staff Papers, n. 9).

KALDOR, N. A model of economic growth. Economic Journal, v. 67, n. 268, p. 591-624, 1957. $\overline{100,1956 .}$

Alternative theories of distribution. Review of Economic Studies, v. 23, n. 2, p. p. 83- 
KALECKI, M. Selected essays on the dynamics of the capitalist economy. Cambridge: Cambridge University Press, 1971.

. The theory of economic dynamics. Londres: Allen $\mathcal{Z}$ Unwin, 1954.

LEWIS, W. A. Economic development with unlimited supplies of labor. The Manchester School of Economic and Social Studies, v. 22, n. 2, p. 139-191, 1954.

LOPES, J. Crises Econômicas na América Latina: Algumas considerações à luz da teoria de M. Kalecki. Edusp: São Paulo, 2001.

LUCAS, JR. R. E. Why doesn`t capital flow from rich to poor countries? American Economic Review Paper and Proceedings, v. 80, n. 2, p. 92-96, 1990.

MARGLIN, S. Growth, Distribution, and Prices. Cambridge, MA: Harvard University Press, 1984.

MARX, K. O Capital. Rio de Janeiro: Civilização Brasileira, 1988.

MUNDELL, R. The appropriate use of monetary and fiscal policy for internal and external stability. Washington, D.C.: IMF, 1962. (International Monetary Fund Staff Papers, n. 9).

OREIRO, J. L. Poupança externa e performance macroeconômica: uma análise a partir de um modelo macrodinâmico não-linear de acumulação de capital e endividamento externo. Revista de Economia Política, v. 24, n. 2, p. 185-200, 2004.

PASINETTI, L. The rate of profit and income distribution in relation to the rate of economic growth. Review of Economic Studies, v. 29, n. 4, p. 267-279, 1962.

ROBINSON, J. A model of accumulation. In: SEN, A. (Org.). Growth Economics. Middlesex: Penguim Books, 1970. Publicado originalmente em 1962.

. The Accumulation of Capital. Macmillan: Londres, 1956.

RODRIK, D. Who needs capital-account convertibility? In: KENEN, P. Should the IMF pursue capital-account convertibility? Princeton, NJ: International Finance Section, Department of Economics, Princeton University, 1998. (Essays in International Finance, n. 207).

ROS, J. Development theory and the economics of growth. Ann Arbor: The University of Michigan Press, 2001.

SIMONSEN, M. H.; CYSNE, R, P. Macroeconomia. São Paulo: Atlas, 1995.

SOLOW, R. A contribution to the theory of economic growth. Quarterly Journal of Economics, v. 70, n. 1, p. 65-94, 1956.

STEINDL, J. Maturity and stagnation in american capitalism. Nova Iorque: Monthly Review Press, 1952.

TAKAYAMA, A. Analytical methods in economics. Michigan: The University of Michigan Press, 1993. 
TAYLOR, L. A stagnationist model of economic growth. Cambridge Journal of Economics, v. 9, n. 2, p. 383-403, 1985.

. Inflation, income distribution, and growth. Cambridge, MA: MIT Press, 1991.

. Reconstructing macroeconomics. Cambridge, MA: Harvard University Press, 2004.

. Varieties of stabilization experience. Oxford: Clarendon Press, 1998.

Recebido em: 26/05/2011. Aceito em: 06/05/2014. 